\title{
EXPERIMENTAL AND THEORETICAL INVESTIGATION ON CORROSION INHIBITION OF MILD STEEL IN SULPHURIC ACID BY COCCINIA INDICA LEAVES EXTRACT
}

\author{
S. Jyothi* and K. Rathidevi \\ Department of Chemistry, Kumaraguru College of Technology, \\ Coimbatore-641049,Tamilnadu (India) \\ *E-mail: jyothi.sci@kct.ac.in
}

\begin{abstract}
Experimental and theoretical investigation on corrosion inhibition of mild steel (MS) in sulphuric acid by Coccinia indica (CI) leaves extract was studied using mass loss and quantum chemical calculation methods. The inhibition efficiency increased with increase in the concentration of the extract but decreased with rising in temperature and increase in acid strength. Furthermore, the inhibition efficiency synergistically increased on the addition of halide ions. The thermodynamic parameter indicates the spontaneous adsorption of the inhibitor on MS surface. The adsorption of CI on MS surface obeyed Langmuir adsorption isotherm. The individual compounds present in the CI extract were identified by GC-MS. FTIR and optical profiler images confirmed the adsorption of CI on MS surface. Quantum chemical studies confirmed that the MS is protected from corrosion by adsorption of the constituents in CI extract.

Keywords: Corrosion inhibitor, steel, surface roughness, thermodynamics, quantum mechanics.

(C) RASĀYAN. All rights reserved

\section{INTRODUCTION}

Corrosion is an inevitable and unavoidable process but it is controllable with suitable measures. Uses of many inhibitors are banned because of their toxicity and the environmental hazards they create. ${ }^{1,2}$ Hence there is a strive to make use of environmentally friendly, non-toxic /less toxic, extracts of naturally occurring plant materials as corrosion inhibitors. Extracts of plant materials contain a wide variety of organic compounds. In recent years volumes of literature are available on the use of plant extracts as corrosion inhibitors for MS in acidic media and they have attracted the attention due to their extended applications. ${ }^{3-5}$ In the search for a natural plant inhibitor, we tumbled upon Coccinia indica (CI), a climber which is available in plenty. The phytochemical database on it suggests that it contains a large number of phytochemical constituents which can be used as corrosion inhibitors. Hence in the present investigation, a focus has been made to investigate the inhibitive effect of an acid extract of CI leaves on the corrosion of mild steel (MS) in the sulphuric acid medium using mass loss method.
\end{abstract}

\section{Materials and Methods}

\section{EXPERIMENTAL}

The experiments were performed with MS with the following composition. (Wt \%) :C 0.143\%, Ni $0.006 \%$, Mo $0.018 \%$, P $0.035 \%$, Si $0.041 \%, \mathrm{Mn} 0.271 \%$ and the rest is Fe. The aggressive solution of $1 \mathrm{~N}$ $\mathrm{H}_{2} \mathrm{SO}_{4}$ was prepared by dilution of $\mathrm{AR}$ grade $97 \% \mathrm{H}_{2} \mathrm{SO}_{4}$ with distilled water. Fresh CI leaves were picked and then cleaned with tap water to eliminate ash and mud, dried in shade and then ground to powder.

\section{Preparation of Extract}

The acid extract was prepared by refluxing $10 \mathrm{~g}$ of dried and milled $\mathrm{CI}$ leaves in $500 \mathrm{ml}$ of $1 \mathrm{~N} \mathrm{H}_{2} \mathrm{SO}_{4}$ for three hours and kept overnight for cooling. It was then filtered and the filtrate was stored in a standard flask. The desired concentrations of the inhibitor were prepared by diluting the above stock solution with distilled water. The concentration range of CI used was 0.2-1.4 (\% v/v). 


\section{GC-MS Analysis}

GC-MS analyses were performed using Instant SHIMADZU GC/MS QP2010 gas chromatographer coupled to a mass spectrometer with highly purified helium as the carrier gas. All the constituents present in the sample were identified in scan mode and quantified in selected ion monitoring (SIM) mode with their characteristic ions by internal standard calibration. Identification of constituents present in CI was done by using Wiley 8th and NIST 08 libraries.

\section{Mass Loss Measurement}

The MS sheet was cut into small pieces of dimensions $1 \times 5 \times 0.15 \mathrm{~cm}$ and were abraded with a series of emery papers, washed with distilled water, degreased with acetone and dried. After weighing accurately specimens in triplicate were immersed in the test solutions for specified periods of immersion in the absence and presence of CI. After a quantified interval of time, the specimens were removed, rinsed with water, dried in the warm air then stored in a desiccator and then reweighed to determine the weight loss. The percentage inhibition efficiency (\% IE), corrosion rate (CR) and surface coverage $(\theta)$ were calculated.

\section{FTIR}

Fourier transform infrared (FTIR) spectra were recorded in Instant SHIMADZU model- IRAFFINITY-1, using the $\mathrm{KBr}$ disk technique.

\section{Surface Analysis}

The specimens used for surface morphology examination were immersed in $1 \mathrm{~N} \mathrm{H}_{2} \mathrm{SO}_{4}$ in the absence and presence of $\mathrm{CI}$ at $27^{\circ} \mathrm{C}$ for 6 hours. The analysis was performed using Zeta-200 optical profiler.

\section{Quantum Chemical Calculations}

The individual compounds present in the CI extract were identified by GC-MS and also retrieved through the survey of the literature. Theoretical calculations and parameters were generated using MOPAC 2007. The structures were first optimized and the simulations are conducted to calculate the electronic parameters. FMO may be used to identify the adsorption centers of the inhibitor molecule. They have also been proved to be a very powerful tool for studying inhibition of the corrosion of metals. ${ }^{6}$ The Quantum chemical calculations were done using Semi-empirical method - Parameterized Model 3 (PM3). ${ }^{7}$ The following quantum chemical parameters were considered: the energies of highest occupied molecular orbital $\left(\mathrm{E}_{\mathrm{HOMO}}\right)$, the lowest unoccupied molecular orbital $\left(\mathrm{E}_{\mathrm{LUMO}}\right)$ and the energy gap $(\Delta \mathrm{E})$.

\section{Components Identification}

\section{RESULTS AND DISCUSSION}

Phytochemical analysis of CI extract shows that it contains rich naturally synthesized biodegradable organic compounds. Lupeol (LP), $\beta$ - Sitosterol (BS), and Stigma-7-en-3- one (SO) have also been isolated. Relative amounts of individual components were calculated on the basis of their GC peak. The analysis of chromatogram shows that there are 24 individual components and of which the following 4 are prominent. Hexadecanoic acid methyl ester (HM), Lupeol, $\beta$ - Sitosterol and Stigma-7-en-3- one.

\section{Mass Loss Measurements}

Table-1 shows the \%IE and $\mathrm{CR}$ for $\mathrm{CI}$ in $1 \mathrm{~N} \mathrm{H}_{2} \mathrm{SO}_{4}$ at room temperature. From the table, it is clear that the concentration of the inhibitor increase, the corrosion rate decrease and the \% IE increase. This indicates that the inhibitor molecules get adsorbed on the metal surface and prevent the further corrosion. The immersion time is another important parameter which ascertains the inhibitive effect of the inhibitor on standing. In the present study, the effect of immersion time (1-6 h) on corrosion inhibition of CI in 1 $\mathrm{N} \mathrm{H}_{2} \mathrm{SO}_{4}$ at room temperature was investigated using mass loss method. From the Table-1 it is clear that as the immersion time is increased from 1-6h, the \% IE values noted at all concentrations of an extract of $\mathrm{CI}$ increase up to $6 \mathrm{~h}$ after that they remain flat. Similarly, the increasing acid strength from 1- $4 \mathrm{~N}$ resulted 
RASĀYAN J. Chem.

Vol. 10 | No. 4 |1253-1260 | October - December | 2017

in decreasing \% IE. This is due to the aggressiveness of the acid. As the concentration of the acid increases, the CR also increases in both inhibited and uninhibited acid. The corrosion rates are observed to be lower in the inhibiting solutions containing extracts-halide ions combination than the extract alone. To study the influence of halide ions on the corrosion inhibition characteristics of an extract of CI leaves in $1 \mathrm{~N} \mathrm{H}_{2} \mathrm{SO}_{4}, 1 \mathrm{ppm}$ of $\mathrm{KCl}, \mathrm{KBr}$ and $\mathrm{KI}$ were added to the test solution. The extract of CI leaves was kept at $1.4 \%(\mathrm{v} / \mathrm{v})$. The result obtained in the present study clearly shows that the inhibition efficiency of the CI was greatly enhanced by the addition of halide ions and could be ascribed to a synergistic effect. The results revealed that the synergistic effect is more pronounced in the presence of $\mathrm{I}^{-}$ than $\mathrm{Br}^{-}$than $\mathrm{Cl}^{-}$. The greater influence of the iodide ion is often attributed to its large ionic radius, high hydrophobicity, and low electronegativity, compared to the other halide ions. ${ }^{8}$

Table-1: Influence of concentration of CI extract on the corrosion of MS in $1 \mathrm{~N} \mathrm{H}_{2} \mathrm{SO}_{4}$

\begin{tabular}{c|c|c|c|c|c|c|c|c|c|c|c|c}
\hline \multirow{2}{*}{$\begin{array}{c}\text { CI } \\
\begin{array}{c}\text { Conc. } \\
(\% \text { v/v })\end{array}\end{array}$} & \multicolumn{2}{|c|}{$1 \mathrm{hr}$} & \multicolumn{2}{c|}{$2 \mathrm{hrs}$} & \multicolumn{2}{c|}{$3 \mathrm{hrs}$} & \multicolumn{2}{c|}{$4 \mathrm{hrs}$} & \multicolumn{2}{|c|}{$5 \mathrm{hrs}$} & \multicolumn{2}{c}{$6 \mathrm{hrs}$} \\
\hline (mpy) & $\begin{array}{c}\text { IE } \\
(\%)\end{array}$ & $\begin{array}{c}\text { CR } \\
(\mathrm{mpy})\end{array}$ & $\begin{array}{c}\text { IE } \\
(\%)\end{array}$ & $\begin{array}{c}\text { CR } \\
(\mathrm{mpy})\end{array}$ & $\begin{array}{c}\text { IE } \\
(\%)\end{array}$ & $\begin{array}{c}\text { CR } \\
(\mathrm{mpy})\end{array}$ & $\begin{array}{c}\text { IE } \\
(\%)\end{array}$ & $\begin{array}{c}\text { CR } \\
(\mathrm{mpy})\end{array}$ & $\begin{array}{c}\text { IE } \\
(\%)\end{array}$ & $\begin{array}{c}\text { CR } \\
(\mathrm{mpy})\end{array}$ & $\begin{array}{c}\text { IE } \\
(\%)\end{array}$ \\
\hline Blank & 353.23 & - & 207.15 & - & 287.82 & - & 312.8 & - & 347.99 & - & 489.77 & - \\
\hline 0.2 & 196.24 & 40.74 & 94.38 & 55.79 & 95.89 & 67.17 & 75.78 & 76.66 & 52.33 & 84.96 & 75.95 & 86.52 \\
\hline 0.4 & 126.47 & 62.96 & 90.31 & 56.84 & 72.68 & 74.74 & 52.33 & 83.28 & 43.61 & 87.47 & 43.73 & 91.11 \\
\hline 0.6 & 122.11 & 65.43 & 78.34 & 62.10 & 60.83 & 78.79 & 43.61 & 85.89 & 40.12 & 88.47 & 38.94 & 92.44 \\
\hline 0.8 & 117.74 & 66.66 & 73.59 & 67.89 & 57.93 & 79.80 & 41.43 & 86.24 & 35.76 & 89.72 & 34.89 & 93.04 \\
\hline 1.0 & 109.02 & 69.14 & 58.53 & 71.58 & 48.35 & 83.08 & 38.16 & 87.80 & 33.14 & 90.48 & 29.80 & 93.43 \\
\hline 1.2 & 91.58 & 74.07 & 48.19 & 76.84 & 45.06 & 84.34 & 37.07 & 88.15 & 31.18 & 90.98 & 23.26 & 95.26 \\
\hline 1.4 & 61.05 & 82.72 & 34.89 & 83.16 & 36.34 & 87.37 & 30.53 & 90.24 & 25.29 & 92.48 & 14.54 & 96.74 \\
\hline
\end{tabular}

\section{Effect of Temperature}

To find out the effect of temperature on the \% IE and CR, mass loss experiments were carried out by varying the temperature from $303-333 \mathrm{~K}$ in the absence and presence of varying concentrations of the extract of CI leaves for an immersion period of one hour and the results obtained are given in the Table- 2 . As the temperature increases from 303-333K, the corrosion rate is increased and the inhibition efficiency of the inhibitor is decreased. This might be due to the fact that increase of temperature affect the rate of adsorption / desorption ratio and the rate of desorption could have been faster than the rate of adsorption, leading to the decrease in inhibition efficiency with temperature. ${ }^{9}$ The decrease in $\%$ IE with increase in temperature generally increases the activation energy of the corrosion process in the presence of the inhibitor. ${ }^{10,11}$

Table-2: Effect of temperature on the corrosion of MS in the presence of various concentrations of CI extract in $1 \mathrm{~N}$ $\mathrm{H}_{2} \mathrm{SO}_{4}$

\begin{tabular}{c|c|c|c|c|c|c|c|c}
\hline \multirow{2}{*}{$\begin{array}{c}\mathrm{CI} \\
\begin{array}{c}\text { Conc. } \\
(\% \text { v/v })\end{array}\end{array}$} & $\begin{array}{c}\text { CR } \\
(\mathrm{mpy})\end{array}$ & $\begin{array}{c}\mathrm{IE} \\
(\%)\end{array}$ & $\begin{array}{c}\mathrm{CR} \\
(\mathrm{mpy})\end{array}$ & $\begin{array}{c}\mathrm{IE} \\
(\%)\end{array}$ & $\begin{array}{c}\mathrm{CR} \\
(\mathrm{mpy})\end{array}$ & $\begin{array}{c}\mathrm{IE} \\
(\%)\end{array}$ & $\begin{array}{c}\mathrm{CR} \\
(\mathrm{mpy})\end{array}$ & $\begin{array}{c}\mathrm{IE} \\
(\%)\end{array}$ \\
\hline Blank & 366.32 & - & 409.92 & - & 1613.53 & - & 2572.93 & - \\
\hline 0.2 & 175.79 & 51.19 & 296.54 & 26.09 & 1198.53 & 25.68 & 1962.41 & 23.73 \\
\hline 0.4 & 161.35 & 55.95 & 209.32 & 47.82 & 924.51 & 42.70 & 1583.09 & 40.68 \\
\hline 0.6 & 148.27 & 60.71 & 187.52 & 53.26 & 868.46 & 45.95 & 1439.10 & 44.07 \\
\hline 0.8 & 135.19 & 66.66 & 152.63 & 61.96 & 750.16 & 56.76 & 1201.05 & 52.54 \\
\hline 1.0 & 122.11 & 69.04 & 143.90 & 64.13 & 702.10 & 58.11 & 1090.23 & 57.63 \\
\hline 1.2 & 109.02 & 70.24 & 126.47 & 68.48 & 671.59 & 58.65 & 1046.62 & 59.32 \\
\hline 1.4 & 82.85 & 77.38 & 122.11 & 70.65 & 603.17 & 62.70 & 1003.01 & 61.01 \\
\hline
\end{tabular}


RASĀYAN J. Chem.

Vol. 10 | No. 4 |1253-1260 | October - December | 2017

It is also clear from the results that the \% IE decreases with increase in temperature. Temperature studies help in the calculation of thermodynamic parameters like the free energy of adsorption $\left(\Delta \mathrm{G}_{\mathrm{ads}}\right)$, enthalpy of adsorption $\left(\Delta \mathrm{H}_{\mathrm{ads}}\right)$ and entropy of adsorption $\left(\Delta \mathrm{S}_{\mathrm{ads}}\right)$. Results obtained indicate that the values of $\Delta \mathrm{G}_{\mathrm{ads}}$ are negative in all the cases indicating that both extracts of CI leaves are strongly adsorbed on the metal surface. The negative values also indicate the stability of adsorbed layer and the spontaneous adsorption of the inhibitor molecules on the MS surface. In the present study the negative value of $\Delta \mathrm{H}_{\text {ads }}$ indicates that the adsorption of components of the extracts undergo an exothermic process. ${ }^{10} \Delta S_{\text {ads }}$ values are positive; this behaviour might be explained in the following way: Before the adsorption of inhibitor molecules onto steel surface, inhibitor molecules can randomly move in the bulk solution, however, with the progress of adsorption, inhibitor molecules are orderly adsorbed onto steel surface. ${ }^{11}$

\section{Adsorption Isotherm}

Adsorption isotherms are very important in understanding the mechanism of inhibition of corrosion reactions. The most frequently used adsorption isotherms are Langmuir, Temkin, Freundlich, Frumkin, Flory Huggins, Bockris-Swinkel and El-Awardy isotherms.It has been found that the data fit well with the Langmuir adsorption isotherm with correlation coefficient nearing unity.

\section{FTIR}

FTIR spectra were used to determine the functional groups in the plant extract and analyze the corrosion protective film formed on the MS surface. The comparison of FTIR spectra of CI extract (Fig.-1a) and the adsorption product between the extract and scrapped powder (Fig.-1b) have undergone significant shifts in the FTIR absorption bands for various groups. The following frequency shifts are observed for the CI extract and the solid adsorption product, viz 3543.12 to $3111.50 \mathrm{~cm}^{-1}$ (-OH stretching), 1621.82 to $1633.12 \mathrm{~cm}^{-1}$ (-C=O stretching), 1575 to $1549 \mathrm{~cm}^{-1}(\mathrm{C}=\mathrm{C})$. The above shifts in absorption bands reveal the adsorption of different components of the $C I$ extract on the MS surface. The band at 550-800 $\mathrm{cm}^{-1}$ only found in the scratched coupon probably originates mainly from $\gamma \mathrm{Fe}_{2} \mathrm{O}_{3}$. Thus, the surface analysis using FTIR reveals that functional groups like $-\mathrm{OH},-\mathrm{COOH}, \mathrm{N}-\mathrm{H}, \mathrm{C}=\mathrm{N}, \mathrm{C}=\mathrm{O}$, present in the organic constituents of CI Extract are involved in adsorption on MS via hydrogen bonding and/or weak Van der Waals forces. ${ }^{12}$ Hence, the FTIR spectra studies reveal the interaction of the inhibitor on the MS surface for adsorption in corrosion protection.

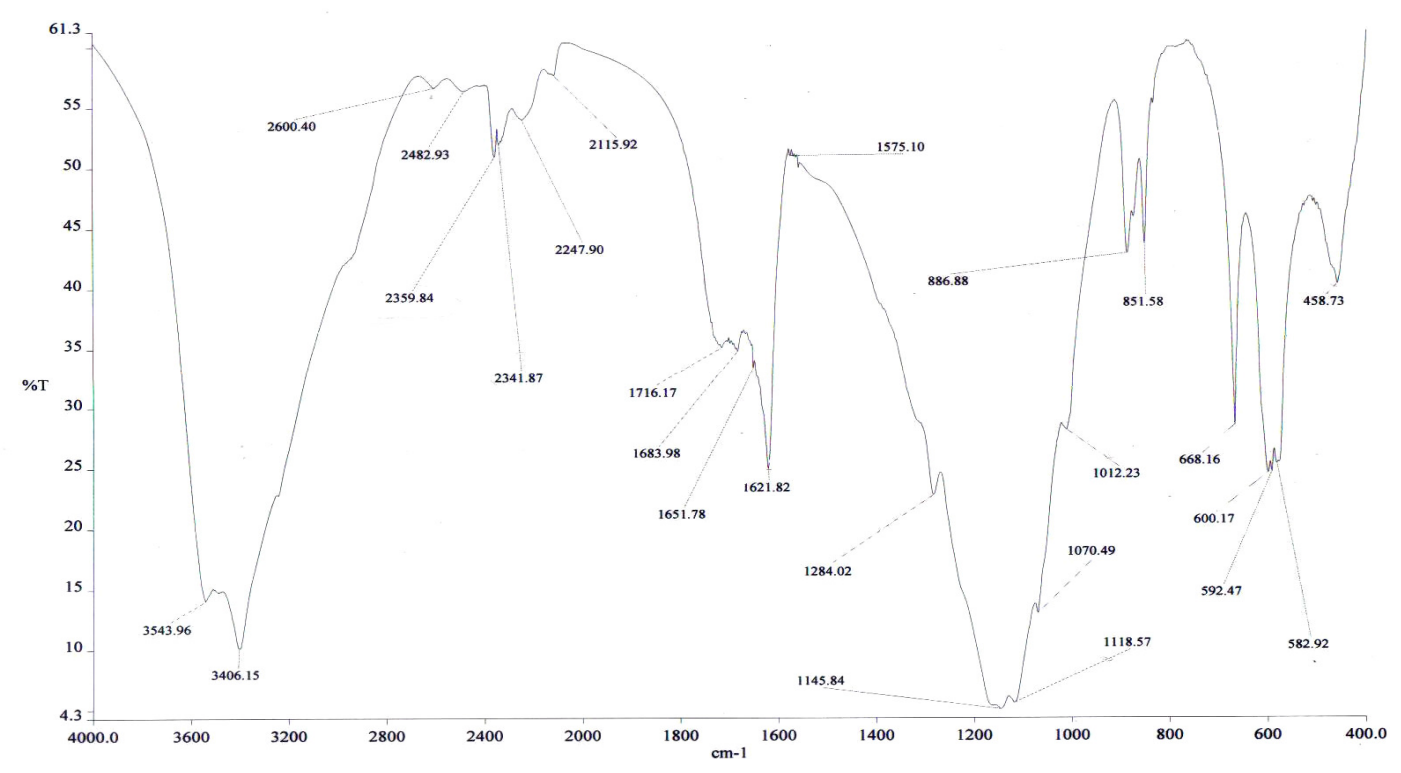

Fig.-1a: FTIR spectrum of $\mathrm{H}_{2} \mathrm{SO}_{4}$ extract of CI concentrates 
RASĀYAN J. Chem.

Vol. 10 | No. 4 |1253-1260 | October - December | 2017

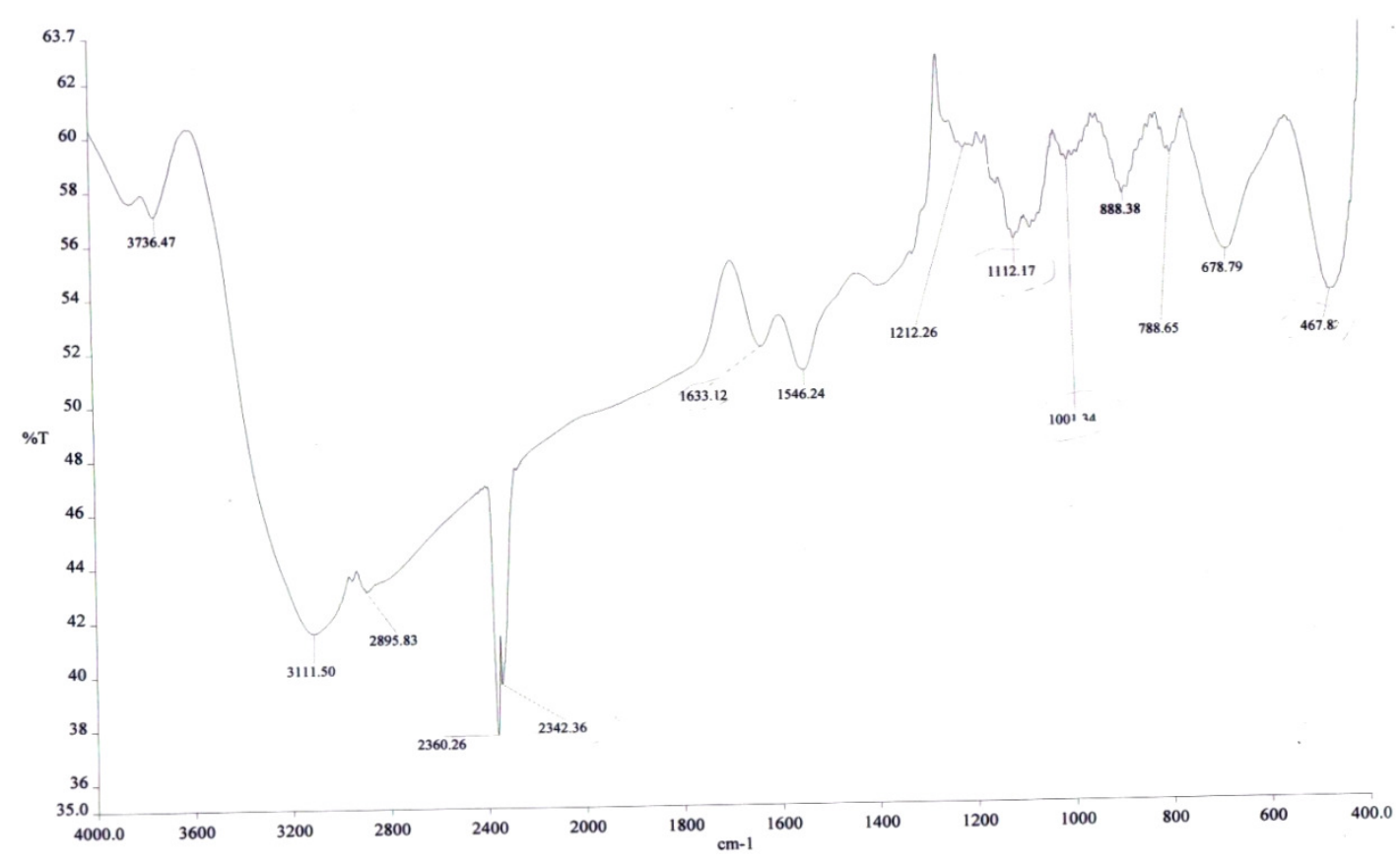

Fig.-1b: FTIR spectrum of the adsorbed material of MS in the presence of $\mathrm{H}_{2} \mathrm{SO}_{4}$ extract of CI

\section{Surface Analysis}

Surface analysis of MS was studied by optical profilometry after $24 \mathrm{~h}$ immersion in $1 \mathrm{~N} \mathrm{H}_{2} \mathrm{SO}_{4}$ both in the absence and the presence of CI extract. Figure-2a represents the micrograph obtained for polished MS without being exposed to the corrosive environment. The corroded metal surface with etched grain boundaries is clearly seen in blank $\mathrm{H}_{2} \mathrm{SO}_{4}$ in Fig.-2b due to the direct attack of aggressive acids. The micrographs of MS in the presence of $\mathrm{CI}$ in $1 \mathrm{~N} \mathrm{H}_{2} \mathrm{SO}_{4}$ is given in Fig.-2c. From these figures, it is clearly evident that a protective film of phytochemical constituents is formed on the surface of MS. This film is responsible for corrosion protection.

\section{Quantum Chemical Calculation}

A theoretical investigation based on quantum chemical calculations is proposed as a powerful tool for predicting a number of molecular parameters directly related to the corrosion inhibiting property of any chemical compound. ${ }^{13,14}$ Quantum chemical calculations are utilized to ascertain whether there is a clear relationship between the molecular structures of the inhibitor and its inhibition effect. In the present work, the structural parameters and adsorptive performance of the major constituents of CI such as Lupeol (LP), $\beta$ - Sitosterol (BS), Stigma-7-en-3- one (SO) and Hexadecanoic acid methyl ester (HM)were used to elucidate the inhibition mechanism. The output of MOPAC program report values is listed in Table-3. The optimized structures, HOMO and LUMO structures of the studied compounds are given in Fig.-3.

Table-3: Calculated Quantum- Chemical Parameters

\begin{tabular}{c|c|c|c}
\hline Compounds & $\mathrm{E}_{\mathrm{HOMO}}$ & $\mathrm{E}_{\mathrm{LUMO}}$ & $\Delta \mathrm{E}$ \\
\hline Lupeol (LP) & -11.93 & 2.94 & 14.87 \\
\hline$\beta$ - Sitosterol (BS) & -12.69 & 11.67 & 24.36 \\
\hline Stigma-7-en-3- one (SO) & -11.57 & -4.10 & 7.47 \\
\hline Hexadecanoic acid methyl ester(HM) & -12.68 & 0.048 & 12.73 \\
\hline
\end{tabular}

Frontier molecular orbital theory is useful in predicting adsorption centers of the inhibitor molecules responsible for the interaction with a surface metal atom. ${ }^{15}$ Moreover, the gap between the LUMO and 


\section{RASĀYAN J. Chem.}

Vol. 10 | No. 4 |1253-1260 | October - December | 2017

HOMO energy level of the molecules is another important factor that should be considered. It is well established in the literature that the higher the HOMO energy of the inhibitor, the greater is the tendency for the donation of electrons to an unoccupied orbital of the metal, and the higher the corrosion inhibition efficiency.
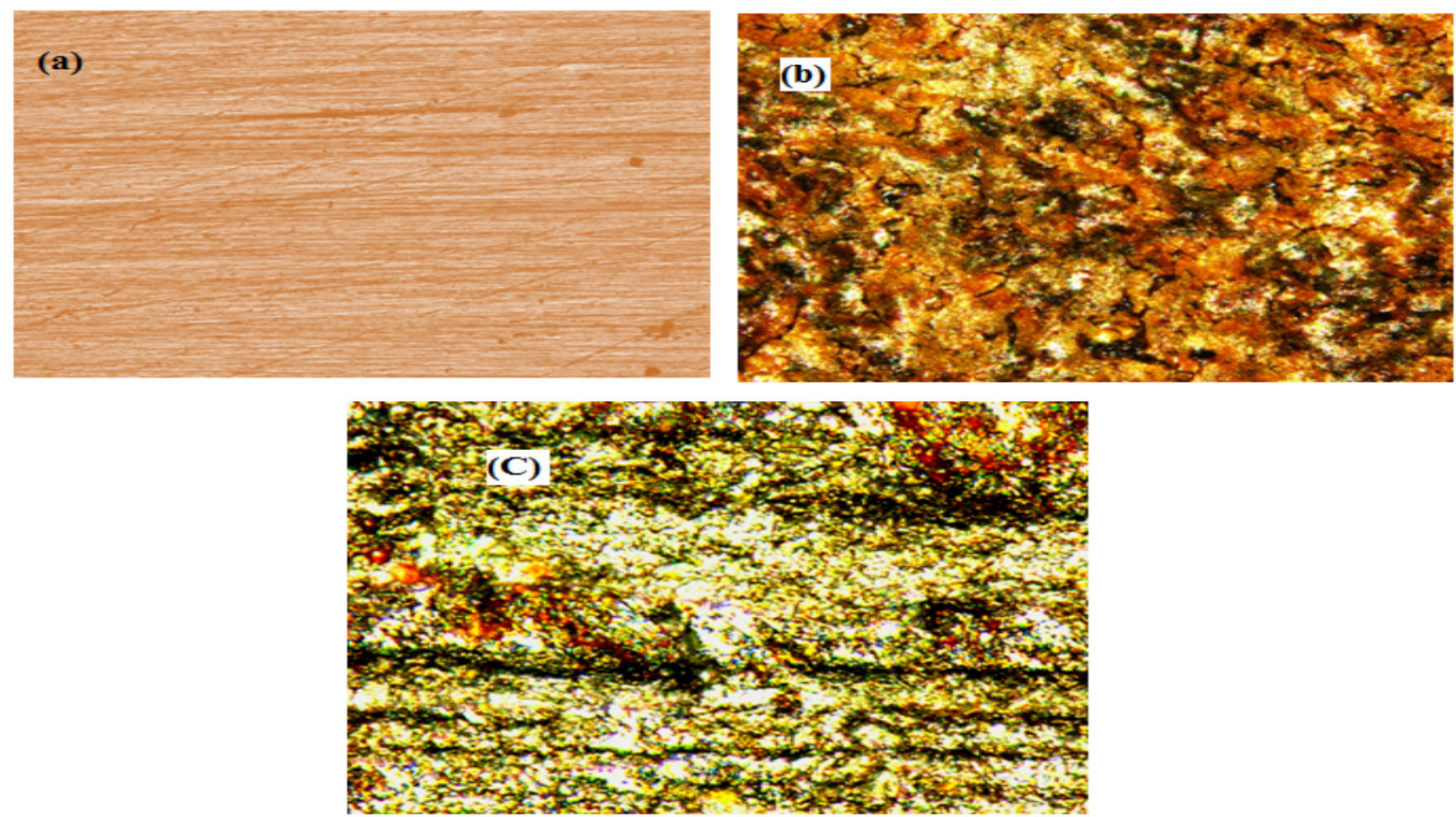

Fig.-2: (a) Optical profiler images of polished MS, (b) in $1 \mathrm{M} \mathrm{H}_{2} \mathrm{SO}_{4}$, (c) With CI extract

(3b) Optimized Geometry




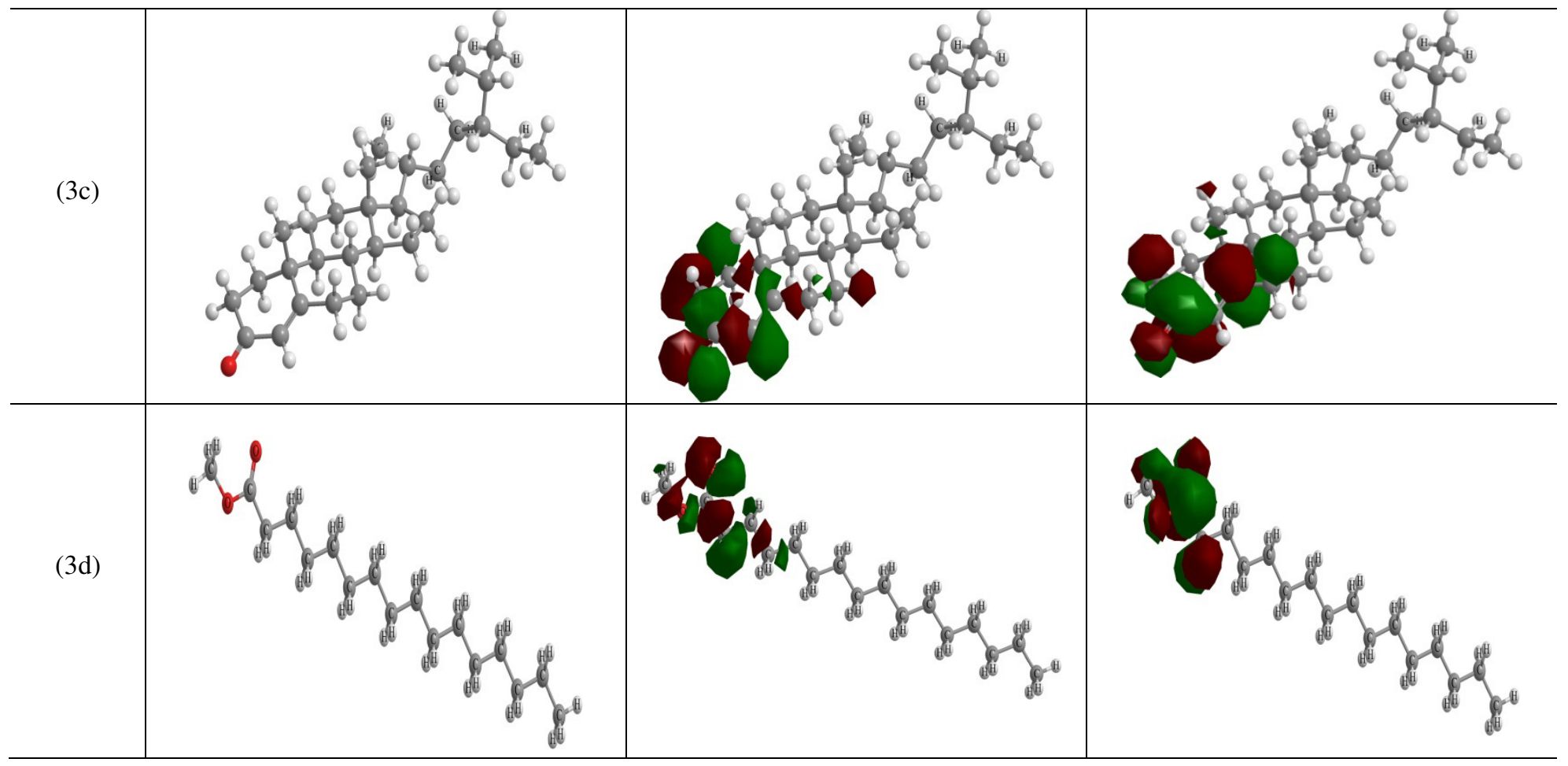

Fig.-3: Optimized, HOMO and LUMO structures of (a) LP, (b) BS, (c) SO and (d) HM

In addition, the lower the LUMO energy, the easier the acceptance of electrons from metal surface thus as the LUMO-HOMO energy gap is decreased, the efficiency of inhibitor is improved. The value of Еномо, $\mathrm{E}_{\mathrm{LUMO}}$ and $\Delta \mathrm{E}$ of $\mathrm{SO}$ indicates the better inhibition efficiency. A molecule with a low energy gap is more polarizable and is generally associated with the high chemical activity and low kinetic stability and is termed as a soft molecule. In our study, the trend for the $(\Delta \mathrm{E})$ values follow the order $\mathrm{SO}<\mathrm{LP}<\mathrm{HM}<$ $\mathrm{BS}$, which suggests that SO has the highest reactivity in comparison to the other compounds and would therefore likely interact strongly with the metal surface. Thus quantum chemical calculations are useful in pinpointing the fact that $\mathrm{SO}$ is most efficient over others.

\section{CONCLUSION}

The CI extract acts as good and efficient corrosion inhibitor for MS in $1 \mathrm{~N} \mathrm{H}_{2} \mathrm{SO}_{4}$. The inhibition efficiency values increase with increasing $\mathrm{CI}$ concentrations, time and in the presence of halide ions and decrease with temperature. The adsorption of CI on MS surface obeys Langmuir adsorption isotherm. FTIR spectroscopic studies reveal that the phytochemical constituents of the CI extract are adsorbed on the surface of MS resulting in the shift of characteristic adsorption frequencies of the functional groups. Optical profiler studies show a significant improvement in surface morphology in the presence of CI extract in acidic media. Quantum chemical parameters such as HOMO-LUMO energy gap were carried out by DFT which indicates the presence of Stigma-7-en-3- one form a uniform film over the MS surface, which effectively protects from the corrosion.

\section{REFERENCES}

1. H. Al-Sehaibani, Material Wissen Werkstatt Technology, 31, 1060 (2000).

2. A. Chetouani and B. Hammouti, Bulletin of Electrochemistry, 19, 23 (2001).

3. K. Krishnaveni, J. Ravichandran and A. Selvaraj, Acta Metallurgica Sinica (English letters) 26, 321(2013).

4. A.Y. El-Etre, Journal of Colloid and Interface Science, 314,578 (2007). 
RASĀYAN J. Chem.

Vol. 10 | No. 4 |1253-1260 | October - December | 2017

5. P. C. Okafor, M.E. Ikpi, I. E. Uwah, E.E. Ebenso, U.J. Ekpe and S. A. Umoren, Corrosion Science, 50, 2310 (2008).

6. K.C. Emregül and M. Hayvalí , Corrosion Science, 48, 797(2006).

7. J.P. Stewart, Journal of Computational Chemistry,10, 209 (1989).

8. C. Jeyaprabha, S. Sathiyanarayanan, S. Muralidharan, G. Venkatachari, Journal of the Brazilian Chemical Society, 17, 61(2006).

9. G. L. Zacchini, F. Zucchi and G. Trabanelli, In Proceedings of III European Symposium on Corrosion Inhibitors, Ann. Univ. Ferrara, N.S. Sez. V . Supl, pp. 577 (1971).

10. E. Khamis, Corrosion Science, 46(6), 476 (1990).

11. A.Y. El-Etre, Journal of Colloid and Interface Science, 314(2), 578 (2007).

12. L.R. Chauhan and G. Gunasekaran, Corrosion Science, 49(3), 1143(2006).

13. K.F. Khaled and N. Hackerman, Electrochimica Acta, 48, 2715 (2003).

14. M. Bouayed, H. Rabaa, A. Srhiri, J.Y. Saillard, A.B. Bachir and A.L. Beuze, Corrosion Science, 41,501 (1999).

15. J. Fang and J. Li, Journal of Molecular Structure, 593, 179 (2002).

[RJC-1924/2017] 